\title{
Mitochondria-Targeted Antioxidant Peptide SS31 Prevents Hypoxia/ Reoxygenation-Induced Apoptosis by Down-Regulating p66Shc in Renal Tubular Epithelial Cells
}

\author{
Wen-Yu Zhao $\quad$ Shu Han ${ }^{a}$ Lei Zhang You-Hua Zhu Li-Ming Wang Li Zeng \\ Organ Transplantation Institute of PLA, Changzheng Hospital, Second Military Medical University, \\ Shanghai, aThese authors contributed equally to the study
}

\section{Key Words}

SS31 $\bullet$ Apoptosis $\bullet$ p66Shc $・$ Renal tubular epithelial cells

\begin{abstract}
Background/Aims: Ischemia/reperfusion injury plays a crucial role in renal transplantation and represents a significant risk factor for acute kidney injury and delayed graft function. Mitochondria-targeted antioxidant peptide SS31 has been shown to attenuate ischemia/ reperfusion injury by inhibiting oxidative stress. The present study was carried out to investigate whether the pretreatment of SS31 could reduce hypoxia/reoxygenation (H/R)-induced injury by inhibiting p66Shc. Methods: The cultured rat renal proximal tubular cell line NRK52E cells were exposed to $24 \mathrm{~h}$ hypoxia $\left(5 \% \mathrm{CO}_{2}, 1 \% \mathrm{O}_{2^{\prime}} 94 \% \mathrm{~N}_{2}\right.$ ) followed by $6 \mathrm{~h}$ reoxygenation $(5 \%$ $\left.\mathrm{CO}_{2}, 21 \% \mathrm{O}_{2}, 74 \% \mathrm{~N}_{2}\right)$. SS31 was added to the culture medium $4 \mathrm{~h}$ prior to the treatment. Then the cell viability, apoptosis, and oxidative stress levels were determined. In addition, western blot analysis was performed to determine the expression of p66Shc, p-p66Shc, cytochrome c, and caspase-3. Results: H/R induced apoptotic cell death, accompanied with activation of total and p-p66Shc in NRK52E cells. Pretreatment with SS31 or overexpression of a dominantnegative Ser36 mutant p66Shc (p66Shc S36A) or p66Shc siRNA prevented cell death, whereas the protection effect of SS31 was completely blocked by overexpression of wild-type p66Shc. Furthermore, SS31 pretreatment reduced H/R-induced intracellular oxidative stress, cytochrome c translocation to the cytoplasm, and caspase- 3 activation through inhibiting p66Shc. Conclusion: This study revealed that SS31 pretreatment serves a protective role against $\mathrm{H} / \mathrm{R}$-induced apoptosis of human renal tubular epithelial cells, and the mechanism is related to suppression of p66Shc.
\end{abstract}


Zhao/Han/Zhang/Zhu/Wang/Zeng: SS31 Prevents H/R-Induced Apoptosis

\section{Introduction}

Ischemia-reperfusion (I/R) injury is one of the major risk factors for acute kidney injury (AKI) in renal transplantation and the development of chronic allograft nephropathy. AKI remains a major clinical problem associated with considerable morbidity and mortality with little progress made in treatment outcome in decades [1]. The huge unmet medical need in renal I/R injury motivated intensive biomedical research to understand the pathophysiology of I/R injury at the cellular and molecular level, which would facilitate development of effective pharmaceutical interventions.

Renal I/R injury involves complex etiological and pathophysiological processes. During renal I/R, reactive oxygen species (ROS)-mediated damage in renal proximal tubular cells leads to acute tubular necrosis, which is the hallmark of I/R-induced AKI [2-4]. ROS scavengers and antioxidants have been shown to protect against renal I/R injury in various cellular and animal experiments since early 1990s [5]. It is believed that protecting renal proximal tubular cells from ROS-mediated damage by antioxidants is a plausible strategy to prevent and treat renal I/R injury and AKI.

The main source of ROS generated during I/R is the mitochondrial electron transport chain. It has been well established that mitochondrial dysfunction is involved in the pathology for ischemic cell death during kidney transplantation $[6,7]$. p66Shc, an adaptor protein involved in regulation of cellular responses to oxidative stress and life span [8], has recently been shown to mediate mitochondrial dysfunction in renal tubular cells under oxidative stress in vitro [9-11] and renal I/R-induced mitochondrial dysfunction in vivo [12]. p66Shc is phosphorylated at Ser36 under oxidative stress and translocated to the mitochondrial intermembrane space where it binds and oxidizes cytochrome c, leading to excess production of ROS and mitochondrial depolarization [13]. Overexpression of a dominant-negative mutant p66Shc (p66Shc S36A) or p66Shc knockdown with siRNA attenuated or reversed ROS production and apoptosis in renal tubular cells exposed to various external insults [9$12]$, suggesting that p66Shc down-regulation by therapeutic agents might be an effective approach to protect renal tubular cells from I/R injury in kidney transplantation.

SS31 belongs to a family of cell-permeable mitochondria-targeted peptide antioxidants (ss peptides) that was first reported to inhibit ROS production, mitochondrial depolarization, and (I/R)-induced myocardial stunning in early 2000s [14, 15]. SS31 is a tetrapeptide with a dimethyltyrosine residue, which provides ROS scavenging activity [15]. In more recent studies, SS31 has been reported to protect multiple cell types including renal proximal tubular cells against various external insults in vitro and in vivo [16-24] and reduce oxidative stress and attenuate (I/R)-induced myocardial infarction, brain injury, and kidney injury in vivo [25-27]. However, mechanisms underlying the cytoprotective properties of SS31 have not been fully elucidated. In the present study, we investigated whether SS31 protects renal tubular epithelial cell line NRK52E cells against (hypoxia/reoxygenation)-induced apoptosis in vitro and whether the peptide protection effect is p66Shc-dependent.

\section{Materials and Methods}

Reagents

SS-31 was prepared by solid-phase synthesis and provided by Dr. Peter W. Schiller (Clinical Research Institute of Montreal, Montreal, QU, Canada) [28].

Cell culture and treatment

Normal rat renal tubular epithelial cell line NRK52E was originally obtained from ATCC and maintained in Dulbecco's modified Eagle's medium (DMEM) with L-glutamine and 15 mM HEPES supplemented with $10 \%$ fetal bovine serum (FBS), $100 \mathrm{U}$ penicillin, and $100 \mu \mathrm{g} \cdot \mathrm{ml}^{-1}$ streptomycin. Cells were plated into a 96well plate and cultured in a $37^{\circ} \mathrm{C}, 5 \% \mathrm{CO}_{2}$ humidified incubator until confluency. Various concentrations of 
Zhao/Han/Zhang/Zhu/Wang/Zeng: SS31 Prevents H/R-Induced Apoptosis

SS31 were added $4 \mathrm{~h}$ before exposure to H/R. Cells were devided into four treatment groups: (1) Control: cells were incubated under normoxic conditions $\left(5 \% \mathrm{CO}_{2}, 21 \% \mathrm{O}_{2}\right.$, and $74 \% \mathrm{~N}_{2}$ ) without SS31 treatment; (2) SS31: cells were treated with SS31 and incubated under normoxic conditions; (3) H/R: cells were exposed to $24 \mathrm{~h}$ of hypoxia $\left(5 \% \mathrm{CO}_{2}, 1 \% \mathrm{O}_{2}\right.$, and $\left.94 \% \mathrm{~N}_{2}\right)$ followed by $6 \mathrm{~h}$ of reoxygenation $\left(5 \% \mathrm{CO}_{2}, 21 \% \mathrm{O}_{2}\right.$, and $74 \% \mathrm{~N}_{2}$ ). (4) H/R + SS31: cells pretreated with SS31 were exposed to $24 \mathrm{~h}$ of hypoxia followed by $6 \mathrm{~h}$ of reoxygenation.

\section{Cell viability and apoptosis assays}

Cell viability was determined by MTT assay according to the method of Mosmann [29]. Briefly, $5 \mathrm{mg} /$ $\mathrm{ml}$ MTT in phosphate buffer solution (PBS) was added to culture medium. After $4 \mathrm{~h}$ incubation at $37^{\circ} \mathrm{C}$, culture medium was removed, $200 \mu \mathrm{l}$ dimethyl sulfoxide was added, and solubilized formazan product was measured by absorbance at $580 \mathrm{~nm}$. Results were expressed as percent (\%) of absorbance at $580 \mathrm{~nm}$, assuming that the absorbance of the control group was 100\%. Apoptosis was assessed by double-staining with fluorescein (FITC) labeled annexin V and propidium iodide (PI) as previously described [30]. Briefly, 5 $\mu \mathrm{l}$ of annexin V-FITC (Biovision, Milpitas, CA, USA) and $10 \mu \mathrm{l}$ of $50 \mathrm{mg} / \mathrm{ml}$ PI-PBS solution (Sigma, St Louis, MO, USA) were applied to $1 \times 10^{5}$ cells suspended in $500 \mu$ of staining buffer $\left(140 \mathrm{mM} \mathrm{NaCl}, 2.5 \mathrm{mM} \mathrm{CaCl}_{2}\right.$, 10 mM HEPES; pH 7.4). After 15 min incubation in the dark at room temperature, cells were immediately subject to analysis on a flow cytometer. Data acquisition was completed within $1 \mathrm{~h}$ to avoid cell damage and PI diffusion. Results were interpreted using Cellquest software.

\section{Western blotting}

Proteins from the total cellular content and specific cellular compartments (cytoplasm and mitochondria) were analyzed by western immunoblotting using $\beta$-actin as an internal control for equal protein loading. Proteins were separated on 10\% SDS-PAGE and transferred to nitrocellulose membranes. Membranes were blocked by $1 \mathrm{~h}$ incubation at room temperature in Tris-buffered saline solution (TBS-T: $20 \mathrm{mM}$ Tris, $\mathrm{pH}$ 7.6, $135 \mathrm{mM} \mathrm{NaCl}, 0.05 \%$ Tween) containing 5\% nonfat dry milk. Membranes were then incubated with primary antibodies against p66Shc, p-p66Shc, Cyt.C, Caspase 3, mHSP70 and $\beta$-actin (Santa Cruz Biotechnology, Santa Cruz, CA, USA) at $4^{\circ} \mathrm{C}$ overnight. After three washes with TBS-T, membranes were incubated with horseradish peroxidase-labeled IgG (Boster Biotech, Wuhan, China) for $2 \mathrm{~h}$ at room temperature. Membranes were again washed three times with TBS-T and then with TBS, and subject to detection by SuperSignal western blotting kits from Pierce Biotech (Rockford, IL, USA). Protein levels were interpreted by densitometric analysis using Quantity One1 Software (v4.5.2) from Bio-Rad (Hercules, CA, USA).

\section{Plasmid construction and transient transfection}

Plasmids encoding wild-type p66shc and p66shc S36A mutant [31], and p66Sch small interfering RNA (p66Sch siRNA) [9] were prepared according to reported methods and transiently transfected into NRK52E cells using Lipofectamine LTX reagent from Invitrogen (Grand Island, NY, USA) in six-well plates. Overexpression and knockdown of p66shc were assessed by western blotting.

\section{Detection of ROS and malondialdehyde (MDA)}

Intracellular ROS level was determined according to method described by Wang and Joseph [32]. NRK52E cells were seeded in 24-well plates $\left(5 \times 10^{4}\right.$ per well) and incubated with $10 \mathrm{mM}$ of 2', $7^{\prime}$-dichlorofluorescein diacetate (DCFH-DA; Beyotime, Jiangsu, China) for $30 \mathrm{~min}$ at $37^{\circ} \mathrm{C}$ in the dark. Cells were washed with pre-warmed serum-free DMEM at least three times and cellular fluorescence at 485 $\mathrm{nm}_{\text {exc }} / 530 \mathrm{~nm}_{\mathrm{em}}$ was measured on an inverted fluorescence microscope (TE-2000U, Nikon). ROS production was expressed as fold to the control group. MDA concentration in culture medium was measured using a lipid peroxidation MDA assay kit from Jiancheng Bioengineering Institute (Nanjing, China) according to manufacturer's instructions. The MDA-thiobarbituric acid adduct (MDA-TBA) was measured by absorbance at $544 \mathrm{~nm}$ on a POLARstar OPTIMA microplate reader from BMG Labtech (Offenburg Germany). Cellular protein was measured using Coomassie brightblue (CBBG250) stain method. MDA production was expressed as nmole/mg protein. 
A

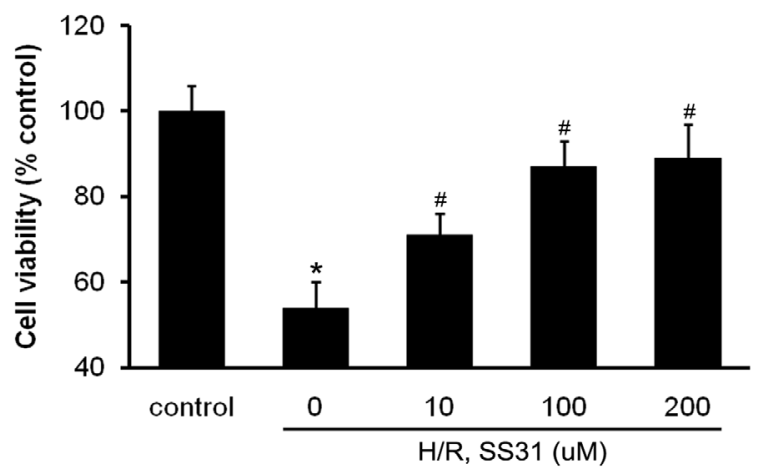

B
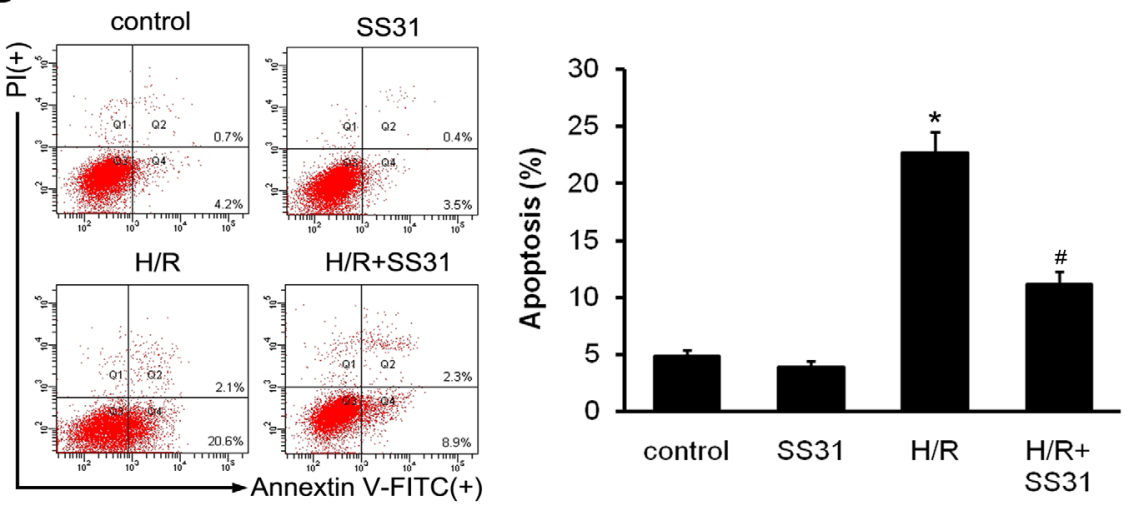

Fig. 1. SS31 inhibits H/R-induced apoptosis in NRK52E cells. (A) Cells were preincubated with SS31 $(0,10,100,200 \mu \mathrm{M})$ for $4 \mathrm{~h}$ prior to $\mathrm{H} / \mathrm{R}$ treatment. Cell viability was determined by MTT assay. Results are expressed as mean \pm SD from three independent experiments. ${ }^{*} P<0.05$ vs. control group, ${ }^{\#} P<0.05$ vs. 0 group. (B) Cells were preincubated with $100 \mu \mathrm{M} \mathrm{SS31}$ for $4 \mathrm{~h}$ prior to H/R treatment. Quantitative assessment of apoptotic cells was performed by annexin V-FITC/PI staining. Results are expressed as mean \pm SD from three independent experiments. ${ }^{*} P<0.05$ vs. control group, ${ }^{\#} P<0.05$ vs. H/R group.

\section{Statistical analysis}

All values were expressed as mean \pm S.D. (standard deviation). Statistical differences were interpreted by one-way analysis of variance (ANOVA). Differences with $P<0.05$ were considered statistically significant. All statistical analyses were conducted using SPSS 13.0 (Chicago, IL, USA) statistical software package.

\section{Results}

SS31 inhibits H/R-induced apoptosis in NRK52E cells

NRK52E cells were exposed to $24 \mathrm{~h}$ hypoxia followed by $6 \mathrm{~h}$ reoxygenation. Compared to the control group, the cell viability was significantly decreased after $\mathrm{H} / \mathrm{R}$ treatment, and this effect was improved by pretreatment with SS31 (Fig. 1A). Among the concentrations tested, $100 \mu \mathrm{M}$ SS31 showed the optimal effect, improving cell viability by more than $82 \%$. Thus, the subsequent experiments were performed with $100 \mu \mathrm{M} \mathrm{SS31}$. Furthermore, quantitative assessment of cell apoptosis was performed on a flow cytometer by Annexin V-FITC/PI staining. As shown in Fig. 1B, the percentage of apoptotic cells increased to 23\% in cells exposed to H/R treatment. Pretreatment of cells with $100 \mu \mathrm{M} \mathrm{SS31} 4 \mathrm{~h}$ prior to H/R treatment attenuated cell apoptosis to about 10\%, indicating that SS31 peptide protected NRK52E cells from H/R-induced cell apoptosis in vitro. 


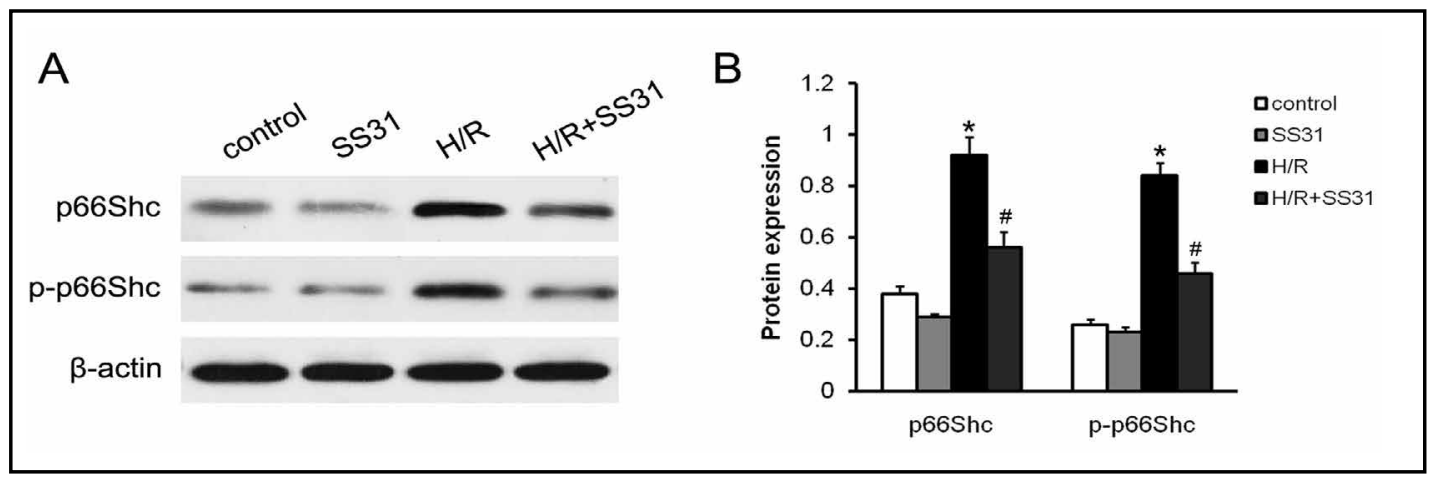

Fig. 2. SS31 prevents H/R-mediated induction of p66Shc in NRK52E cells. (A) Cells were preincubated with $100 \mu \mathrm{M}$ SS31 for $4 \mathrm{~h}$ prior to H/R treatment. The expression of p66Shc and p-p66Shc was analyzed by western blot with $\beta$-actin as the internal control for equal protein loading. (B) Quantification of p66Shc and p-p66Shc expression by densitometry. Results are expressed as mean \pm SD from three independent experiments. ${ }^{*} P<0.05$ vs. control group, ${ }^{\#} P<0.05$ vs. H/R group.

Fig. 3. p66Shc siRNA and p66Shc S36A prevent H/Rinduced apoptosis in NRK52E cells. (A) Western blot analysis of p66Shc and p-p66Shc expression in NRK52E cells. (B) Quantification of p66Shc and p-p66Shc expression by densitometry. Results are expressed as mean \pm SD from three independent experiments. ${ }^{*} P$ $<0.05$ vs. control group, ${ }^{\#} P<$ 0.05 vs. control siRNA group. (C) Cell viability by MTT assay. Results are expressed as mean \pm SD from three independent experiments. ${ }^{*} P<0.05$ vs. H/R group or $\mathrm{H} / \mathrm{R}+$ control siRNA group. (D) Cell apoptosis

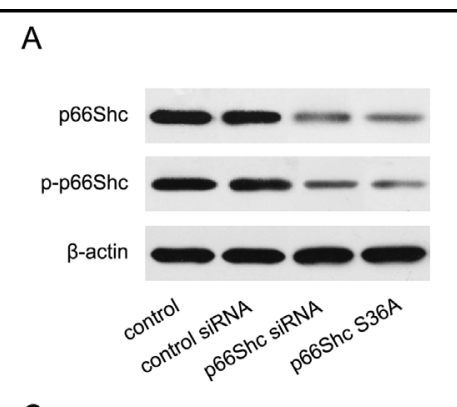

C

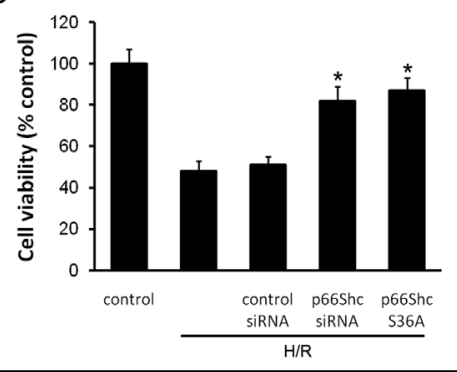

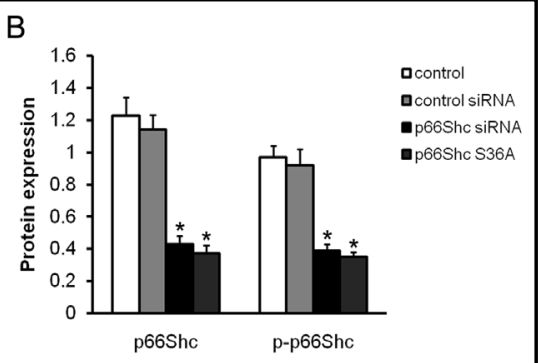

D

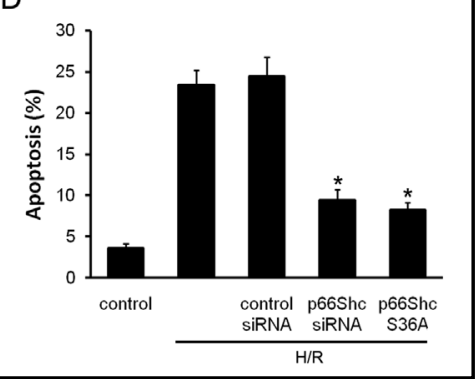

by annexin V-FITC/PI staining. Results are expressed as mean \pm SD from three independent experiments. ${ }^{*} P<0.05$ vs. $\mathrm{H} / \mathrm{R}$ group or $\mathrm{H} / \mathrm{R}+$ control siRNA group.

SS31 prevents $H / R$-mediated induction of p66Shc in NRK52E cells

Expressions of total p66Shc and p66Shc phosphorylated at Ser36 (p-p66Shc) were evaluated by western blotting. As shown in Fig. 2A and B, total p66Shc and p-p66Shc were detected at low levels in control NRK52E cells, and their levels were dramatically increased in cells after H/R treatment. Pretreatment with $100 \mu \mathrm{M}$ SS31 significantly attenuated total p66Shc and p-p66Shc levels after H/R.

SS31 inhibits $H / R$-induced apoptosis by down-regulating p66Shc expression

Because we observed that SS31 down-regulated p66Shc/p-p66Shc expression after H/R in NRK52E cells, we carried out experiments to find out whether p66Shc was directly involved in the protective effect of SS31 against H/R-induced apoptosis. We generated NRK52E cells transfected with p66Shc siRNA, a dominant-negative mutant p66Shc (p66Shc S36A), or wild type p66Shc (WT-p66Shc). Down-regulation of p66Shc by transfection with 

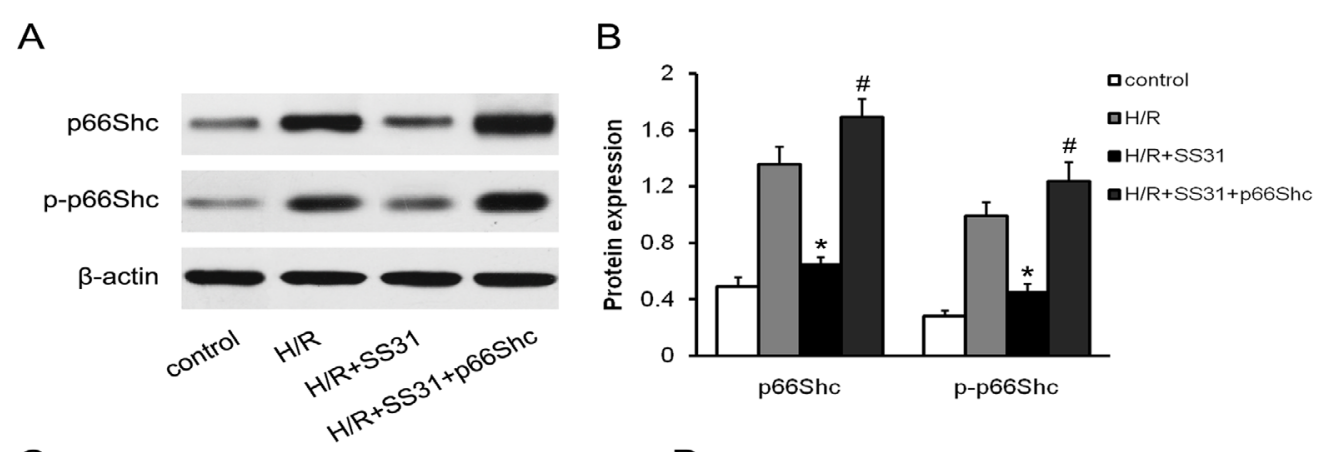

C

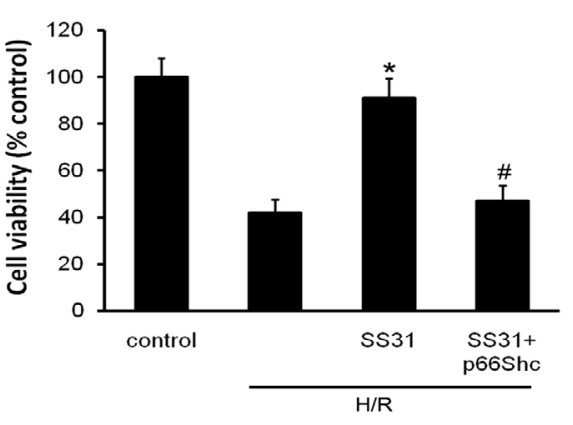

D

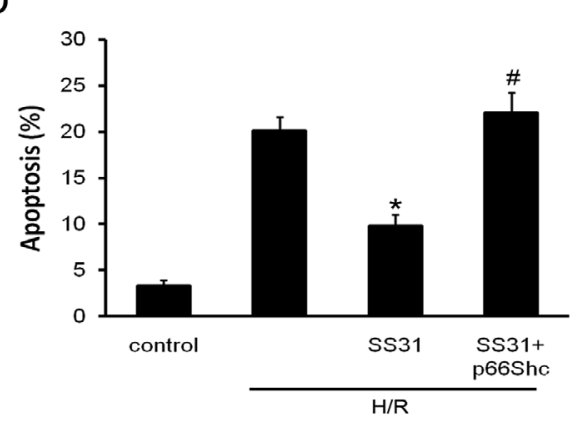

Fig. 4. Overexpression of p66Shc reverses the inhibitory effect of SS31 on H/R-induced cell apoptosis in NRK52E cells. (A) Western blot analysis of p66Shc and p-p66Shc expression in NRK52E cells. (B) Quantification of p66Shc and p-p66Shc expression by densitometry. Results are expressed as mean \pm SD from three independent experiments. ${ }^{*} P<0.05$ vs. H/R group, ${ }^{\#} P<0.05$ vs. H/R + SS31 group. (C) Cell viability by MTT assay. Results are expressed as mean \pm SD from three independent experiments. ${ }^{*} P<0.05$ vs. H/R group, ${ }^{\#} P<0.05$ vs. H/R + SS31 group. (D) Cell apoptosis by annexin V-FITC/PI staining. Results are expressed as mean \pm SD from three independent experiments. ${ }^{*} P<0.05$ vs. H/R group, ${ }^{\#} P<0.05$ vs. H/R + SS31 group.

siRNA or mutant p66Shc S36A was confirmed by western blot analysis (Fig. 3A and 3B). We found that NRK52E cells transfected with siRNA or p66Shc S36A were much more resistant to H/R-induced cell death and apoptosis compared with non-transfected cells and cells transfected with vector alone (Fig. 3C and 3D), indicating that down-regulation of p66Shc provided protection against H/R-induced injury similar to SS31 treatment. Most importantly, overexpression of WT-p66Shc in NRK52E cells completely blocked the protective effect of SS31 against H/R-induced cell apoptosis and total cell death (Fig. 4C and D), suggesting that the protective effect of SS31 was mediated by suppressingp66Shc. Furthermore, our western blot analysis showed that while SS31 decreased p66Shc/p-p66Shc expression after H/R in non-transfected cells, it did not inhibit p66Shc/p-p66Shc in cells overexpressing WT-p66Shc (Fig. 4A and B), verifying that the inhibitory effect of SS31 was p66Shc-dependent.

\section{SS31 reduced H/R-mediated oxidative stress in NRK52E cells}

The effect of SS31 on H/R-induced oxidative stress was assessed by measurement of cellular ROS and MDA levels. As shown in Fig. 5A and B, H/R treatment caused a greater than two times increase in the cellular ROS level and a greater than three times increase in cellular MDA content, signaling severe oxidative stress in H/R-treated cells. In comparison with non-transfected cells, NRK52E cells overexpressing mutant p66Shc S36A were much more resistant to H/R-induced ROS and MDA generation. Pretreatment with $100 \mu$ M SS31 significantly attenuated H/R-induced oxidative stress in NRK52E cells as shown in reduced ROS and MDA levels. Importantly, the protective effect of SS31 against H/R-induced oxidative stress was completely blocked by overexpression of WT-p66Shc, suggesting that SS31 protected NRK52E cells from oxidative stress by down-regulating p66Shc. 


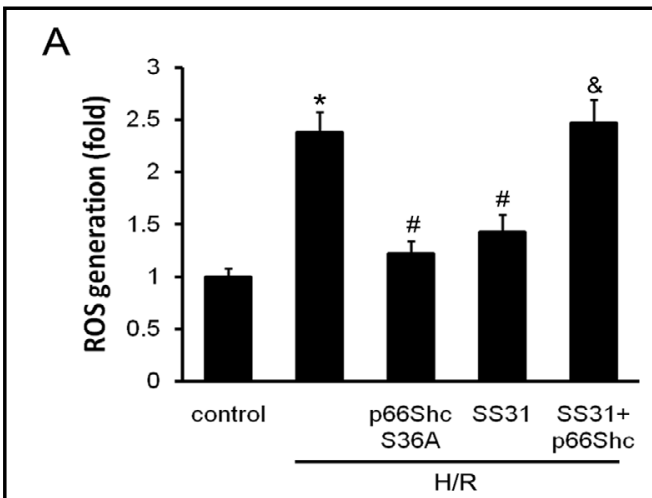

B

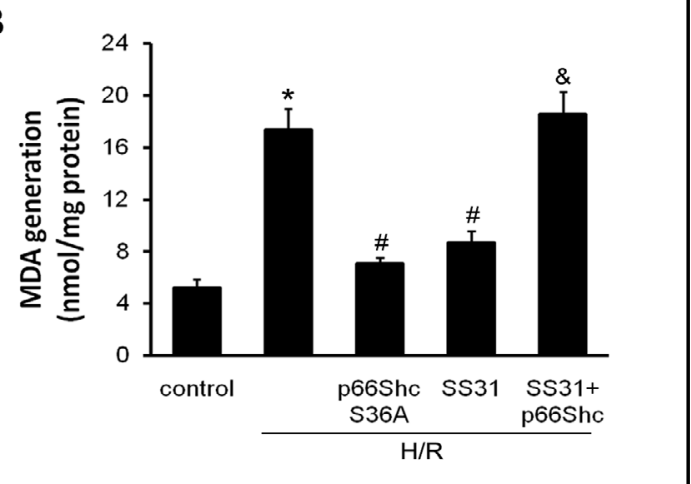

Fig. 5. SS31 inhibits H/R-induced ROS and MDA generation in NRK52E cells. (A) Intracellular ROS levels estimated using the probe DCFH-DA. (B) Cellular MDA levels measured by the TBA method. Concentrations of MDA were in $\mathrm{nmol} / \mathrm{mg}$ protein. Results are expressed as mean $\pm \mathrm{SD}$ from three independent experiments. ${ }^{*} P<0.05$ vs. control group, ${ }^{~} P<0.05$ vs. H/R group, ${ }^{\&} P<0.05$ vs. H/R + SS31 group.

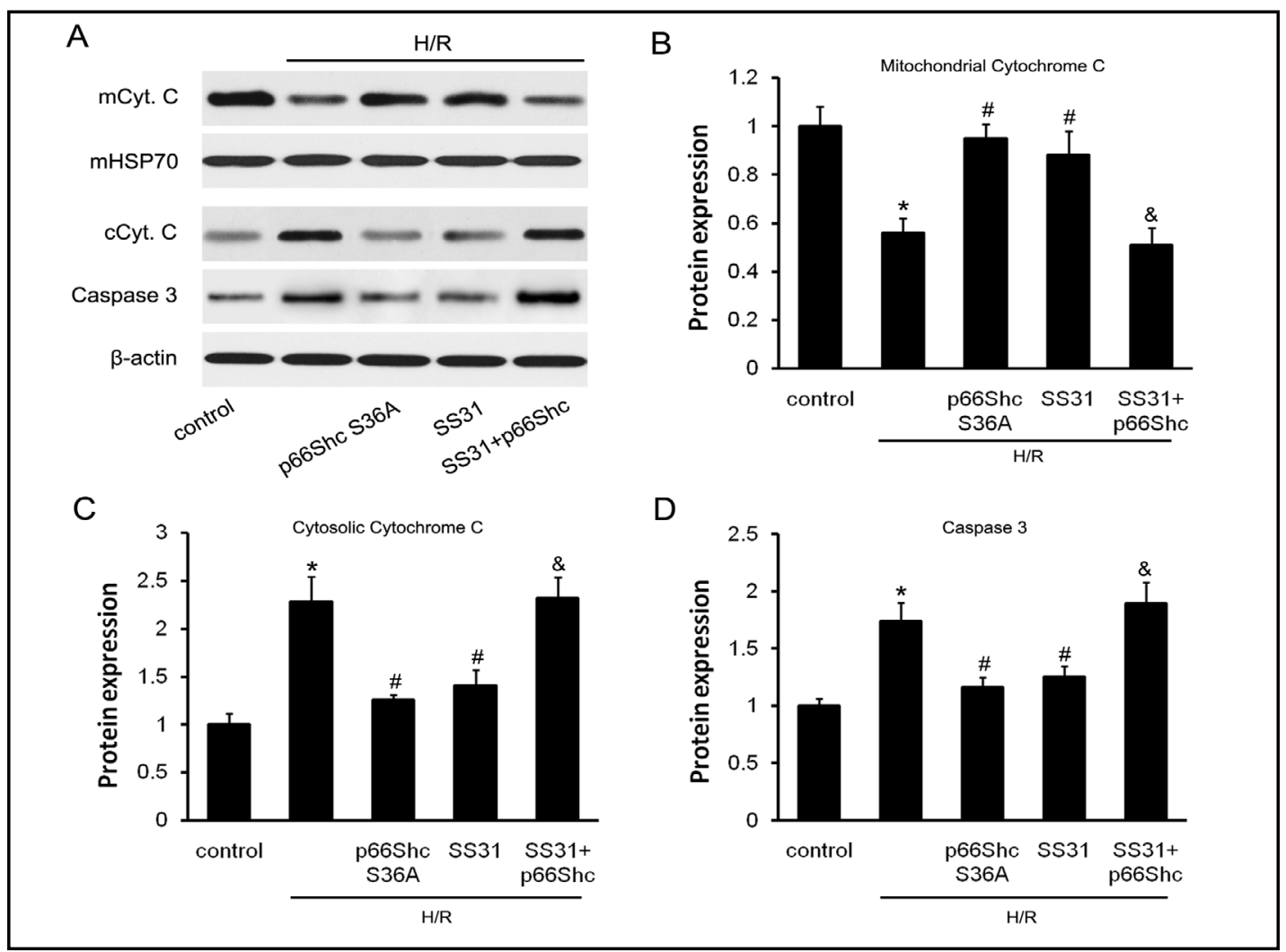

Fig. 6. SS31 inhibits H/R-induced cytochrome c release and caspase-3 activation in NRK52E cells. (A) cytochrome c levels in mitochondria and cytoplasmic compartments and cytosolic caspase-3 levels were assessed by western blot analysis. Mitochondrial heat shock protein 70 (mHSP70) or $\beta$-actin served as loading controls. (B-D) Quantification of protein expression by densitometry: (B) mitochondrial cytochrome c, (C) cytoplasmic cytochrome $c$, and (D) caspase-3. Results are expressed as mean \pm SD from three independent experiments. ${ }^{*} P<0.05$ vs. control group, ${ }^{\sharp} P<0.05$ vs. H/R group, ${ }^{\circledR} P<0.05$ vs. H/R + SS31 group.

\section{SS31 suppresses $H / R$-induced cytochrome c release and caspase-3 activation}

We also examined the effect of SS31 on H/R-induced cytochrome c release and caspase-3 activation, two key events in ROS-induced apoptotic signaling. As shown in Fig. $6 \mathrm{~A}-\mathrm{C}$, cytochrome c mostly resided in mitochondria in control cells under normal culture 
conditions. H/R treatment caused about a 50\% drop in the level of mitochondrial cytochrome $c$ and an over two-fold increase in the level of cytoplasmic cytochrome c, indicating substantial cytochrome $\mathrm{c}$ release following H/R treatment. H/R-treated cells also had much higher caspase-3 level compared with control cells (Fig. 6A and D), indicating activation of the caspase cascade. In comparison with non-transfected cells, NRK52E cells overexpressing mutant p66Shc S36A were much more resistant to H/R-induced cytochrome c release and caspase-3 activation. Pretreatment with $100 \mu \mathrm{M}$ SS31 significantly attenuated H/R-induced cytochrome c release and caspase-3 activation (Fig. 6). Importantly, these protective effects of SS31 were completely blocked by overexpression of WT-p66Shc, providing further evidence that SS31 protected NRK52E cells against H/R-induced apoptosis via p66Shc-dependent pathways.

\section{Discussion}

Reactive oxygen species (ROS) play a critical role in renal I/R injury. ROS generated during I/R cause cell damage not only via direct action on renal cells but also via activation of inflammatory pathways leading to renal inflammation [33,34]. Antioxidants and antiinflammatory compounds are the most intensively exploited pharmaceutical agents against I/R injury and AKI [1, 35]. SS31 is the first ROS scavenger that targets mitochondria and acts upstream of the stress during reperfusion [15]. SS31 has been shown to reduce oxidative stress and attenuate external insult-induced injury in a wide variety of cell types including neurons, hepatocytes, cardiomyocytes, islet cells, and renal tubular cells [9-12]. Treatment with SS31 has also been shown to reduce (I/R)-induced heart, brain, and kidney injury in animal models [25-27]. In particular, Szeto et al. reported that SS31 treatment prevented renal tubular apoptosis and necrosis, reduced oxidative stress and inflammation, and accelerated proliferation of surviving tubular cells in a rat model of renal I/R injury, implying therapeutic potential of SS31 for treatment of ischemic kidney injury [27].

In the present study, we tested the effect of SS31 treatment on H/R-stressed human renal tubular cell line NRK52E cells. We assessed cell injury using viability and apoptosis assays and measured cellular oxidative stress in terms of ROS and MDA levels. MDA is the end product of lipid peroxidation and considered to be an indicator of oxidative stress. In addition, cytochrome $\mathrm{c}$ release and caspase- 3 activation, two established markers for apoptosis, were examined by western blot analysis. We found that exposure to $24 \mathrm{~h}$ hypoxia followed by $6 \mathrm{~h}$ reoxygenation (H/R) significantly increased cell apoptosis and reduced cell viability, boosted ROS and MDA levels, induced cytochrome c translocation from mitochondria to cytoplasm, and activated caspase-3. Pretreatment of NRK52E cells with SS31 $4 \mathrm{~h}$ prior to $\mathrm{H} / \mathrm{R}$ treatment prevented H/R-induced cell death in a dose dependent manner. SS31 also significantly attenuated H/R-induced cell apoptosis, ROS and MDA generation, cytochrome c release, and caspase- 3 activation. Our results demonstrated that SS31 reduced oxidative stress and protected human renal tubular NRK52E cells from ischemic injury in vitro, which was in agreement with previous results observed in a renal I/R injury model in vivo [27].

Although it has been shown that the dimethyltyrosine residue of SS31 was responsible for its radical scavenger activity [15], the mechanisms and pathways involved in the protective effect of SS31 against I/R injury are not fully understood. Cho et al. reported that SS31 attenuated ischemic brain injury by down-regulating CD36, a class B scavenger receptor reported to mediate tissue injury in cerebral ischemia [25]. I/R injury occurs in various clinical and surgical conditions such as myocardial infarction, stroke, and organ transplantation and involves complex pathological processes at the cellular and molecular level, which are largely organ and disease specific [36]. Several recent reports have identified adaptor protein p66Shc as a key mediator in oxidative stress-induced injury in renal tubular cells [9-12]. In the present study, we found that H/R-treatment drastically increased p66Shc/ p-p66Shc expression in NRK52E cells and the protective effect of SS31 against H/R-induced cell death was accompanied by down-regulation of p66Shc/p-p66Shc expression after H/R, 
suggesting that SS31 acted via regulating p66Shc. We also found that overexpression of a dominant-negative Ser36 mutant p66Shc (p66Shc S36A) or p66Shc siRNA could prevent H/R-induced cell death, similar to SS31 treatment. Importantly, the protection effect of SS31 against H/R-induced cell death, oxidative stress, cytochrome c release, and caspase- 3 activation were all completely blocked by overexpression of wild type p66Shc, indicating that p66Shc acted downstream of SS31 and protection by SS31 was p66Shc-dependent.

In conclusion, our results suggest that pharmaceutical intervention of p66Shc signaling either by therapeutic agents such as SS31 or genetic modulation holds promise for prevention and treatment of renal I/R injury. Additional studies in animal models are required to verify and extend our findings.

\section{Acknowledgements}

This study was supported by National Natural Science Foundation of China (No.81000310), Medicine Guide Project of Shanghai Science and Technology Committee (No.114119a8000) and National High Technology Research and Development Program of China (No.2012AA021001).

\section{References}

1 Rouschop KM, Leemans JC: Ischemia-reperfusion treatment: opportunities point to modulation of the inflammatory response. Kidney Int 2008;73:1333-1335.

-2 Venkatachalam MA, Bernard DB, Donohoe JF, Levinsky NG: Ischemic damage and repair in the rat proximal tubule: differences among the S1, S2, and S3 segments. Kidney Int 1978;14:31-49.

3 Paller MS: The cell biology of reperfusion injury in the kidney. J Investig Med 1994;42:632-639.

4 Nath KA, Norby SM: Reactive oxygen species and acute renal failure. Am J Med 2000;109:665-678.

5 Greene EL, Paller MS: Oxygen free radicals in acute renal failure. Miner Electrolyte Metab 1991;17:124132.

76 Jassem W, Fuggle SV, Rela M, Koo DD, Heaton ND: The role of mitochondria in ischemia/reperfusion injury. Transplantation 2002;73:493-499.

7 Hall AM, Unwin RJ: The not so 'mighty chondrion': emergence of renal diseases due to mitochondrial dysfunction. Nephron Physiol 2007;105:1-10.

8 Migliaccio E, Giorgio M, Mele S, Pelicci G, Reboldi P, Pandolfi PP, Lanfrancone L, Pelicci PG: The p66shc adaptor protein controls oxidative stress response and life span in mammals. Nature 1999;402:309-313.

-9 Arany I, Faisal A, Nagamine Y, Safirstein RL: p66shc inhibits pro-survival epidermal growth factor receptor/ERK signaling during severe oxidative stress in mouse renal proximal tubule cells. J Biol Chem 2008;283:6110-6117.

10 Arany I, Faisal A, Clark JS, Vera T, Baliga R, Nagamine Y: p66SHC-mediated mitochondrial dysfunction in renal proximal tubule cells during oxidative injury. Am J Physiol Renal Physiol 2010;298:F1214-1221.

11 Sun L, Xiao L, Nie J, Liu FY, Ling GH, Zhu XJ, Tang WB, Chen WC, Xia YC, Zhan M, Ma MM, Peng YM, Liu H, Liu YH, Kanwar YS: p66Shc mediates high-glucose and angiotensin II-induced oxidative stress renal tubular injury via mitochondrial-dependent apoptotic pathway. Am J Physiol Renal Physiol 2010;299:F1014-1025.

12 Arany I, Clark J, Reed DK, Juncos LA: Chronic nicotine exposure augments renal oxidative stress and injury through transcriptional activation of p66shc. Nephrol Dial Transplant 2013;28:1417-1425.

13 Giorgio M, Migliaccio E, Orsini F, Paolucci D, Moroni M, Contursi C, Pelliccia G, Luzi L, Minucci S, Marcaccio M, Pinton P, Rizzuto R, Bernardi P, Paolucci F, Pelicci PG: Electron transfer between cytochrome c and p66Shc generates reactive oxygen species that trigger mitochondrial apoptosis. Cell 2005;122:221-233.

14 Wu D, Soong Y, Zhao GM, Szeto HH: A highly potent peptide analgesic that protects against ischemiareperfusion-induced myocardial stunning. Am J Physiol Heart Circ Physiol 2002;283:H783-791.

15 Zhao K, Zhao GM, Wu D, Soong Y, Birk AV, Schiller PW, Szeto HH: Cell-permeable peptide antioxidants targeted to inner mitochondrial membrane inhibit mitochondrial swelling, oxidative cell death, and reperfusion injury. J Biol Chem 2004;279:34682-34690. 
Zhao/Han/Zhang/Zhu/Wang/Zeng: SS31 Prevents H/R-Induced Apoptosis

16 Whiteman M, Spencer JP, Szeto HH, Armstrong JS: Do mitochondriotropic antioxidants prevent chlorinative stress-induced mitochondrial and cellular injury? Antioxid Redox Signal 2008;10:641-650.

17 Manczak M, Mao P, Calkins MJ, Cornea A, Reddy AP, Murphy MP, Szeto HH, Park B, Reddy PH: Mitochondriatargeted antioxidants protect against amyloid-beta toxicity in Alzheimer's disease neurons. J Alzheimers Dis 2010;20:S609-631.

18 Calkins MJ, Manczak M, Mao P, Shirendeb U, Reddy PH: Impaired mitochondrial biogenesis, defective axonal transport of mitochondria, abnormal mitochondrial dynamics and synaptic degeneration in a mouse model of Alzheimer's disease. Hum Mol Genet 2011;20:4515-4529.

19 Reddy TP, Manczak M, Calkins MJ, Mao P, Reddy AP, Shirendeb U, Park B, Reddy PH: Toxicity of neurons treated with herbicides and neuroprotection by mitochondria-targeted antioxidant SS31. Int J Environ Res Public Health 2011;8:203-221.

20 Cao M, Jiang J, Du Y, Yan P: Mitochondria-targeted antioxidant attenuates high glucose-induced P38 MAPK pathway activation in human neuroblastoma cells. Mol Med Rep 2012;5:929-934.

-21 Andersson DC, Fauconnier J, Yamada T, Lacampagne A, Zhang SJ, Katz A, Westerblad H: Mitochondrial production of reactive oxygen species contributes to the $\beta$-adrenergic stimulation of mouse cardiomycytes. J Physiol 2011;589:1791-1801.

22 Li J, Chen X, Xiao W, Ma W, Li T, Huang J, Liu X, Liang X, Tang S, Luo Y: Mitochondria-targeted antioxidant peptide SS31 attenuates high glucose-induced injury on human retinal endothelial cells. Biochem Biophys Res Commun 2011;404:349-356.

-23 Mizuguchi Y, Chen J, Seshan SV, Poppas DP, Szeto HH, Felsen D: A novel cell-permeable antioxidant peptide decreases renal tubular apoptosis and damage in unilateral ureteral obstruction. Am J Physiol Renal Physiol 2008;295:F1545-1553.

24 Thomas DA, Stauffer C, Zhao K, Yang H, Sharma VK, Szeto HH, Suthanthiran M: Mitochondrial targeting with antioxidant peptide SS-31 prevents mitochondrial depolarization, reduces islet cell apoptosis, increases islet cell yield, and improves posttransplantation function. J Am Soc Nephrol 2007;18:213-222.

25 Cho S, Szeto HH, Kim E, Kim H, Tolhurst AT, Pinto JT: A novel cell-permeable antioxidant peptide, SS31, attenuates ischemic brain injury by down-regulating CD36. J Biol Chem 2007;282:4634-4642.

26 Cho J, Won K, Wu D, Soong Y, Liu S, Szeto HH, Hong MK: Potent mitochondria-targeted peptides reduce myocardial infarction in rats. Coron Artery Dis 2007;18:215-220.

27 Szeto HH, Liu S, Soong Y, Wu D, Darrah SF, Cheng FY, Zhao Z, Ganger M, Tow CY, Seshan SV: Mitochondriatargeted peptide accelerates ATP recovery and reduces ischemic kidney injury. J Am Soc Nephrol 2011;22:1041-1052.

28 Schiller P, Nguyen T, Berezowska I, Dupuis S, Weltrowska G, Chung N, Lemieux C: Synthesis and in vitro opioid activity profiles of DALDA analogues. Eur J Med Chem 2000,35:895 -901.

29 Mosmann T: Rapid colorimetric assay for cellular growth and survival: application to proliferation and cytotoxicity assays. J Immunol Methods 1983;65:55-63.

30 Brind AM, Hurlstone A, Edrisinghe D, Gilmore I, Fisher N, Pirmohamed M, Fryer AA: The role of polymorphisms of glutathione S-transferases GSTM1, M3, P1, T1 and A1 in susceptibility to alcoholic liver disease. Alcohol Alcohol 2004;39:478-483.

-31 Faisal A, Kleiner S, Nagamine Y: Non-redundant role of Shc in Erk activation by cytoskeletal reorganization. J Biol Chem 2004;279:3202-3211.

-32 Wang H, Joseph JA: Structure-activity relationships of quercetin in antagonizing hydrogen peroxideinduced calcium dysregulation in PC12 cells. Free Radic Biol Med 1999;27:612-616.

-33 Araujo M, Welch WJ: Oxidative stress and nitric oxide in kidney function. Curr Opin Nephrol Hypertens 2006;15:72-77.

-34 Vajdovich P: Free radicals and antioxidants in inflammatory processes and ischemia-reperfusion injury. Vet Clin North Am Small Anim Pract 2008;38:31-123, v. doi: 10.1016/j.cvsm.2007.11.008.

35 Chatterjee PK: Novel pharmacological approaches to the treatment of renal ischemia-reperfusion injury: a comprehensive review. Naunyn Schmiedebergs Arch Pharmacol 2007;376:1-43.

-36 Kalogeris T, Baines CP, Krenz M, Korthuis RJ: Cell biology of ischemia/reperfusion injury. Int Rev Cell Mol Biol 2012;298:229-317. 